\title{
Pediatric meningioma with rhabdoid features developed at the site of skull fracture: illustrative case
}

\author{
Sho Takata, MD, ${ }^{1}$ Akira Tamase, MD, PhD, ${ }^{1}$ Yasuhiko Hayashi, MD, PhD, ${ }^{1}$ Osamu Tachibana, MD, PhD, ${ }^{1}$ Katsuaki Sato, MD, $\mathrm{PhD},{ }^{2}$ and \\ Hideaki lizuka, MD, $\mathrm{PhD}^{1}$ \\ ${ }^{1}$ Department of Neurosurgery, Kanazawa Medical University, Ishikawa, Japan; and ²Department of Diagnostic Pathology, Noto General Hospital, Ishikawa, Japan
}

BACKGROUND Pediatric meningiomas are rare, and only a few cases attributed to trauma and characterized by development at the site of bone fracture have been reported. Both pediatric and traumatic meningiomas have aggressive characteristics.

OBSERVATIONS An 11-year-old boy who sustained a head injury resulting from a left frontal skull fracture 8 years previously experienced a convulsive attack. Imaging revealed a meningioma in the left frontal convexity. Total removal of the tumor with a hyperostotic section was successfully achieved. Intraoperative investigation showed tumor invasion into the adjacent frontal cortex. Histologically, the surgical specimen revealed a transitional meningioma with brain invasion and a small cluster of rhabdoid cells. This led to a final pathological diagnosis of an atypical meningioma with rhabdoid features. The postoperative course was uneventful, and no recurrence of the tumor was found after 2 years without adjuvant therapy.

LESSONS This is the first report of a pediatric meningioma with rhabdoid features occurring at the site of a skull fracture. Meningiomas that contain rhabdoid cells without malignant features are not considered to be as aggressive as rhabdoid meningiomas. However, the clinical course must be carefully observed for possible long-term tumor recurrence.

https://thejns.org/doi/abs/10.3171/CASE21107

KEYWORDS meningioma; pediatric; rhabdoid features; skull fracture; growth

Meningiomas account for approximately $20 \%$ of intracranial tumors and typically occur in adults. Pediatric meningiomas are rare, and their occurrence is reported to occur in $2.2 \%$ of all intracranial meningiomas. They tend to be associated with atypical locations, higher histopathological grades, and aggressive behaviors. $^{1}$

Tumorigenesis of a meningioma after head trauma has been deliberated upon for many years. ${ }^{2-4}$ In 1938, post-traumatic meningioma was first described by Cushing and Eisenhardt, who reported a causal relationship between head trauma characterized by a skull fracture and the subsequent development of a meningioma. ${ }^{2}$ Head trauma with a fracture around the suture can cause considerable damage to the arachnoid cells and/or cause meningeal irritation under the dura mater. This damage facilitates tumor neoplasia of the arachnoid cells in the meningioma. ${ }^{2,5}$ In recent reports of post-traumatic meningiomas, histological findings of the tumors revealed a high incidence of cellular atypia as an aggressive

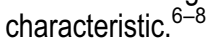

Typically, rhabdoid meningiomas present with malignant characteristics such as local recurrence, remote metastasis, and cerebrospinal fluid dissemination. ${ }^{9,10}$ However, a clinical study at the Mayo Clinic reported that meningiomas with rhabdoid features lacking malignant characteristics are not as aggressive as rhabdoid meningiomas with independent histological features consistent with World Health Organization (WHO) grade III. ${ }^{11}$ Here, we present a case of a post-traumatic meningioma with rhabdoid features lacking other histological characteristics of malignancy, which has not been reported previously.

ABBREVIATIONS CT = computed tomography; $\mathrm{MR}=$ magnetic resonance; $\mathrm{WHO}=$ World Health Organization . INCLUDE WHEN CITING Published October 4, 2021; DOI: 10.3171/CASE21107.

SUBMITTED February 17, 2021. ACCEPTED March 28, 2021.

(c) 2021 The authors, CC BY-NC-ND 4.0 (http://creativecommons.org/licenses/by-nc-nd/4.0/). 


\section{Illustrative Case}

History and Preoperative Assessment

An otherwise healthy 11-year-old boy experienced complex partial seizures during a music class and was transported by ambulance to our hospital. Eight years previously, he had sustained a head injury with a linear skull fracture extending from the anterior skull base to the frontal convexity on the left side (Fig. 1A and B). Computed tomography (CT) revealed an intracranial semicircular mass with a maximum diameter of $4 \mathrm{~cm}$ from the anterior skull base to the frontal convexity (Fig. 1C). The adjacent skull, including the site of the previous linear fracture, was remarkably thickened (Fig. 1D and E). Magnetic resonance (MR) images showed that the mass was extensively adherent to the dura mater with a protrusion into the frontal lobe. The signal intensities demonstrated irregular hypointensity on T1-weighted images and irregular hyperintensity on T2-weighted images (Fig. 2A and B). There was remarkable enhancement of the contrast medium, leading to a radiological diagnosis of meningioma (Fig. $2 \mathrm{C}$ and D).

\section{Operative Course}

Using a frontotemporal craniotomy, we exposed the dura mater of the frontal convexity over the tumor. Under navigation guidance, the dura mater around the dural attachment of the tumor was incised. Soft tumors appeared under the dura mater. Intraoperative microscopic investigation revealed tumor invasion into the adjacent cortex (Fig. 3). Intraoperative histological examination indicated a tentative diagnosis of meningioma with a cluster of rhabdoid cells. We performed total removal of the tumor, including the surrounding cortex, and shaved out the hyperostotic section of the skull. After surgery, the patient recovered well, with no apparent neurological deficits.

\section{Surgical Pathology}

A major section of the tumor was confirmed to be a transitional meningioma (Fig. 4A), and immunohistochemical staining for
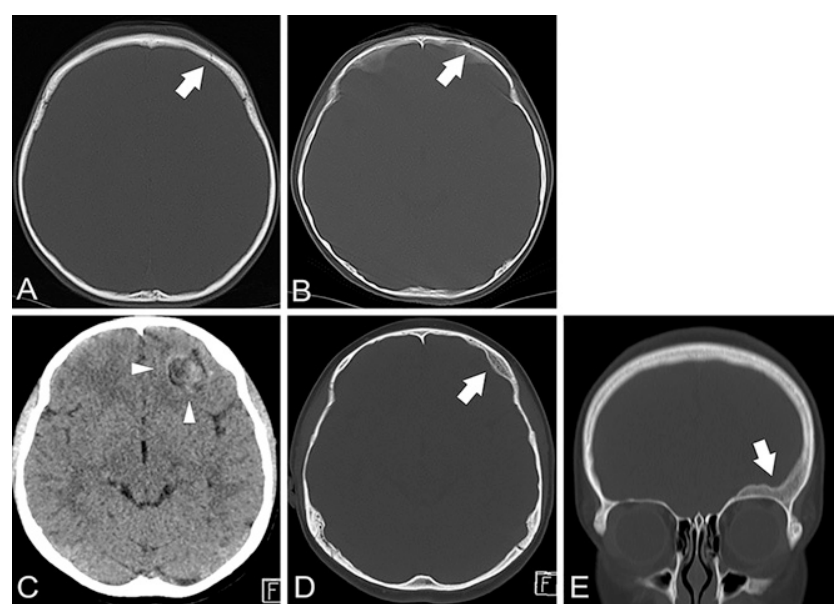

FIG. 1. Axial CT section in the bone window obtained in our hospital revealed a linear skull fracture from the frontal convexity $(\mathbf{A})$ to the anterior skull base (B) on the left side after a head injury when the patient was 3 years old (white arrows). C: Eight years after the injury, a high-density mass was found at the left frontal convexity (arrowheads). Axial (D) and coronal (E) sections of the CT in the bone window clearly show hyperostosis where a previous skull fracture existed (white arrows).
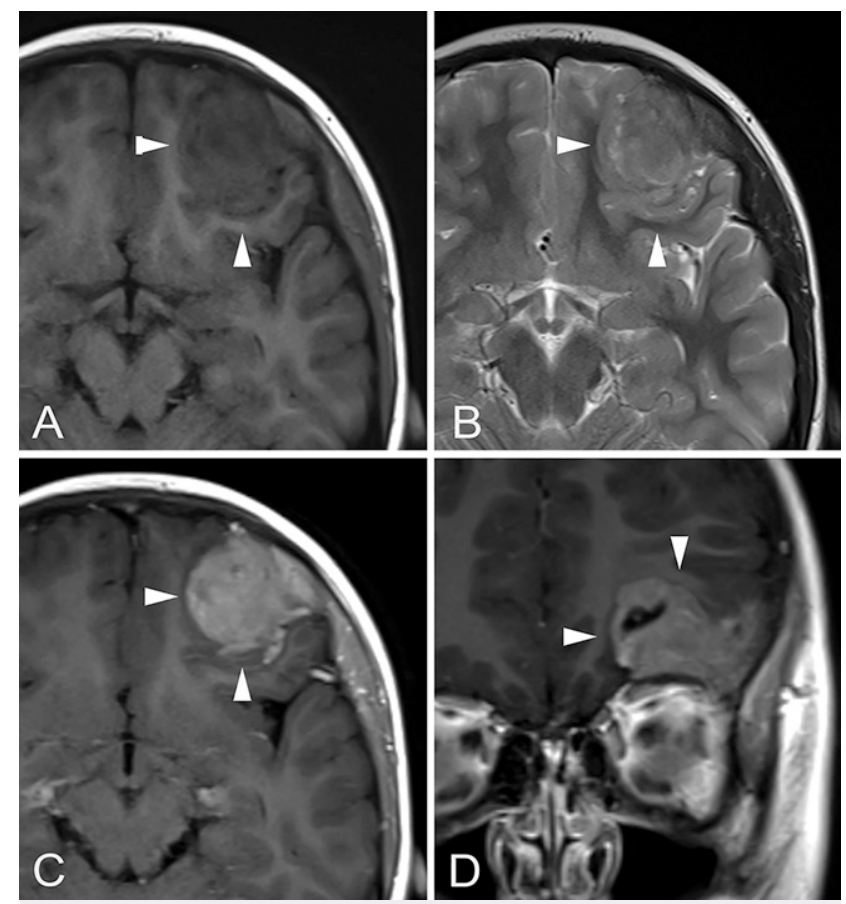

FIG. 2. A round tumor adhered to the dura mater at the left frontal convexity (arrowheads). It was revealed to be an irregular hypointensity on a T1-weighted image $(\mathbf{A})$ with an irregular hyperintensity on a T2-weighted image (B) and had remarkable enhancement after administration of a contrast agent on a T1-weighted image (C). This suggested a meningioma as a radiological diagnosis. The coronal section of a T1-weighted image (D) confirmed that the tumor was extensively connected to the anterior frontal base.

epithelial membrane antigen was strongly positive (Fig. 4B). The MIB-1 index was 5.4\% (Fig. 4C), and mitosis was observed in 1 in 10 high-power fields. Tumor cells invaded the cortex. Cortical regions along the tumor boundary were enriched with psammoma bodies (Fig. 4D). A cluster of rhabdoid cells was characteristically recognized (Fig. 5A); however, they occupied only a small portion of the tumor. The cytoplasm of rhabdoid cells was immunohistochemically positive for vimentin (Fig. 5B). The MIB-1 index was $4.6 \%$ (Fig. 4C). Mitosis was observed in 1 in 10 high-power fields. In addition to rhabdoid morphology, perivascular pseudopapillary features were observed in some areas (Fig. 5D). Histological findings suggestive of malignant characteristics were not observed. Ultimately, we diagnosed an atypical meningioma with rhabdoid features (WHO grade II). The boy was discharged 1 week after the operation, and chronological postdischarge MR images showed no recurrence after 2 years.

\section{Discussion}

\section{Observations}

We present an extremely rare case of a pediatric meningioma that developed at the site of a skull fracture sustained 8 years prior. The characteristic pathological findings of this meningioma consisted of extensive, macroscopically observed invasion into the surrounding cortex, with a cluster of rhabdoid cells without anaplastic features in a microscopically determined transitional meningioma. After total 


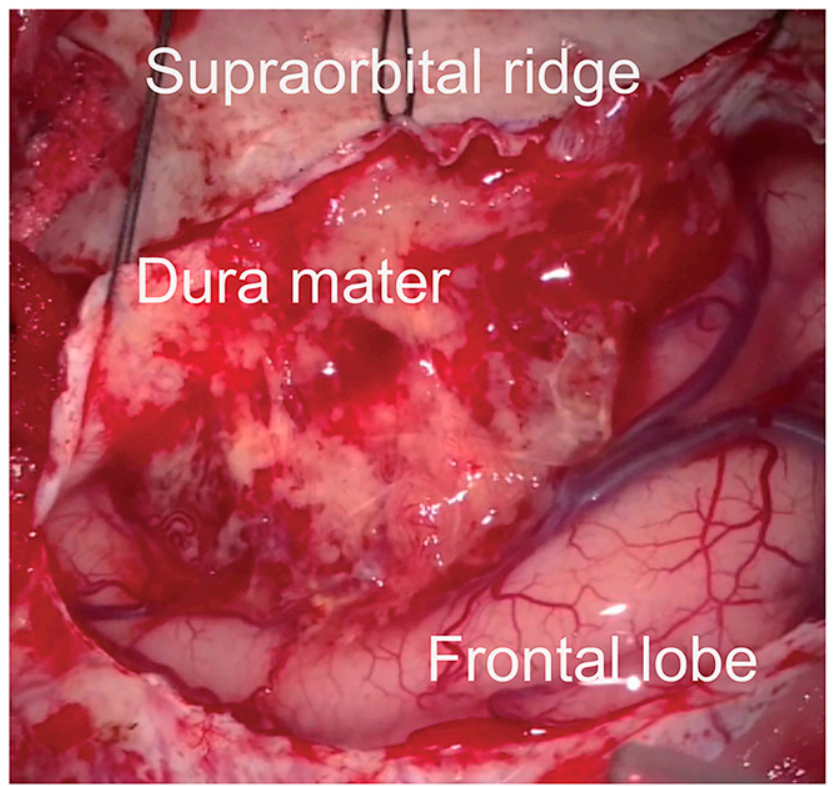

FIG. 3. Intraoperative photo shows that the tumor was firmly adhered to the dura mater from the anterior skull base to the frontal convexity and invaded the adjacent frontal cerebral cortex.

removal of the tumor with the adjacent hypertrophic bone, there has not been a recurrence for 2 years.

Because of the rarity of pediatric meningiomas compared with those occurring in adults, the number of pediatric meningiomas published in the English-language literature is limited to a few cases. $1,12,13$ The frequency of high histological grades, such as
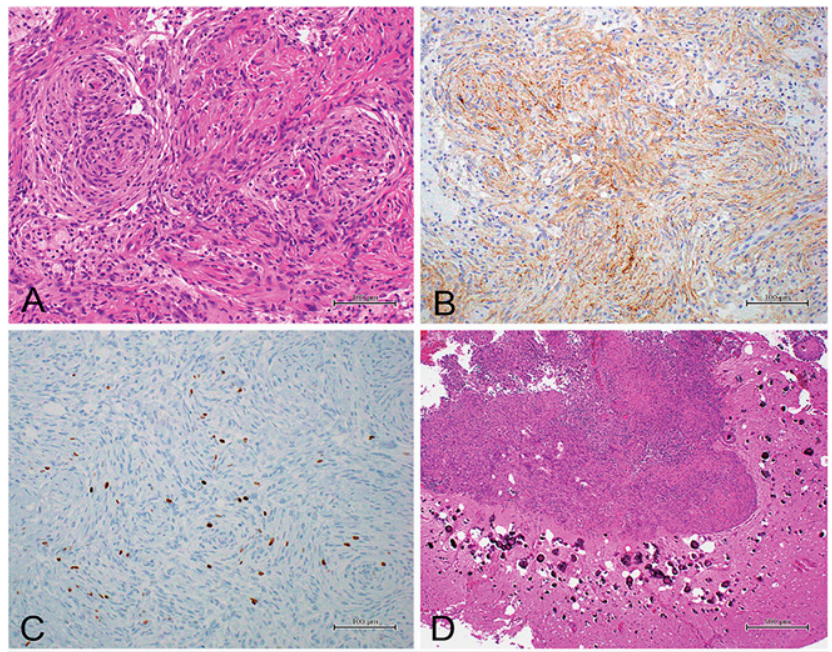

C

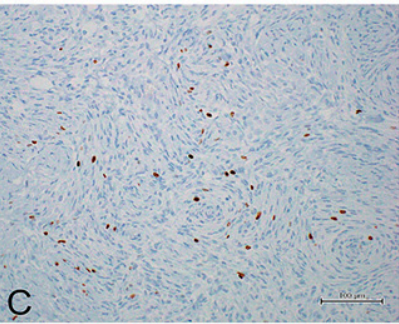

FIG. 4. A: Hematoxylin and eosin staining of the surgical specimens shows that the histological diagnosis was a transitional meningioma (scale bar $=100 \mu \mathrm{m})$. B: Immunohistochemical staining with the antibody for epithelial membrane antigen was strongly positive (scale bar $=100 \mu \mathrm{m}$ ). C: Ki-67 staining showed that the MIB-1 index was as high as $5.4 \%$ (scale bar $=100 \mu \mathrm{m}$ ). D: The meningioma invaded the adjacent cerebral cortex, leading to the diagnosis of an atypical meningioma. Many psammoma bodies along the boundary of the tumor in the cortex were detected (scale bar $=500 \mu \mathrm{m}$ ).

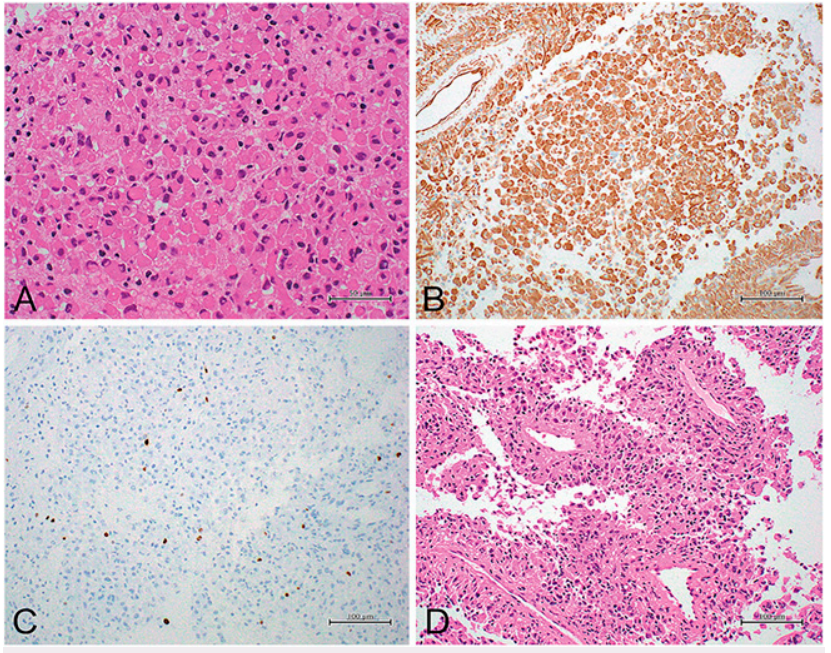

FIG. 5. A: Hematoxylin and eosin staining of the surgical specimen revealed a cluster of rhabdoid cells in the meningioma (scale bar $=$ $50 \mu \mathrm{m})$. B: Vimentin was strongly positive in the cytoplasm of the rhabdoid cells (scale bar $=50 \mu \mathrm{m}$ ). C: Ki-67 staining revealed few mitoses in the rhabdoid cells (scale bar $=50 \mu \mathrm{m}$ ). D: Papillary features were characteristically found even in small sections of the specimen (scale bar $=100 \mu \mathrm{m})$.

WHO grade II or III, accounts for over $30 \%$ of these tumors. ${ }^{12,13}$ Moreover, recurrence in pediatric patients is also more frequent than in adult patients. Among pediatric patients, the recurrence rate of tumors with WHO grade I was found to be not lower than that of tumors with grade II or III, ${ }^{12}$ suggesting that pediatric meningiomas tend to be more aggressive than those in adults. In our case, an aggressive feature was an extensive macroscopic invasion into the surrounding cortex, although no histological malignant features were observed.

Regardless of the limited number of recently published reviews of post-traumatic meningiomas, the reviews support the notion of meningioma development at the site of a cranial fracture ${ }^{6-8}$ Caroli et al. reported that this type of meningioma is more commonly found in the adult population than in the pediatric population. ${ }^{7}$ The period from head trauma to diagnosis was reported to be as long as 13 years on average. A head injury with a severe impact may initiate the development of a meningioma due to the disruption of the arachnoid mater as well as of other structures. ${ }^{7}$ Across all these reviews, the youngest case was a 13-year-old patient. ${ }^{6,7}$ In a few reviews, the most typical histological finding of a post-traumatic meningioma was described as a high frequency of cellular atypia, such as WHO grade II or III, which accounts for approximately $30 \%$ of these tumors. ${ }^{6-8}$ Cushing and Eisenhardt noted that a bone fracture always occurs near a cranial suture and that the meningioma may develop close to the fracture. ${ }^{2}$ Diastasis may result even from a mild head trauma associated with a contusion or laceration of the pachymeninx, which is strongly adherent to the suture. The involvement of arachnoid cells activates the development of the meningioma. ${ }^{2}$ In addition, Barnett et al. suggested that chronic inflammation might lead to meningeal irritation. ${ }^{5}$ As far as we know, the factors provoking the developmental process of post-traumatic meningiomas have not been explored. In our case, the anatomical proximity between the skull fracture and the dural attachment of the 
tumor from the anterior skull base to the frontal convexity was apparent in the evaluation of MR images and bone CT. In addition, the firm attachment of the tumor to the site of the previous fracture was obvious from the intraoperative findings. However, we could not establish a precise causal relationship between the trauma and the development of the meningioma.

Meningiomas associated with rhabdoid features without other histological findings suggesting malignancy are rare pathological entities whose clinical courses have not yet been fully examined. Vaubel et al. reported the results of treatment of 44 patients with rhabdoid meningiomas lacking anaplastic features. ${ }^{11}$ The histological grades of the surgical specimens were WHO grade I $(50 \%)$ or II $(50 \%)$. Recurrence was found in 9 patients (5-year recurrence-free survival, $73.7 \%$ ). The extent of rhabdoid features within the specimens showed high variability and was not significantly associated with recurrence-free survival periods. Therefore, meningiomas that contain rhabdoid cells without malignant features are not considered to be as aggressive as rhabdoid meningiomas with independent histological features consistent with WHO grade III. However, because a few cases leading to death were included in their series, close observation of these patients is essential. Kepes et al. stated, "Rhabdoid features in meningiomas are associated with aggressive biological and clinical behavior." 9 The rates of rhabdoid cells in tumors did not correlate with survival rates, although rhabdoid cell sheets were found to increase in recurrent tumors compared with those in primary tumors. In some cases, the characteristics of rhabdoid meningiomas with papillary features have been reported to exhibit aggressive clinical behavior, such as concomitant atypical or malignant histological characteristics, a higher MIB-1 labeling index, and frequent recurrence. This has led to the classification of a WHO grade III tumor. ${ }^{14,15}$ However, Vaubel et al. noted that even if rhabdoid meningiomas without histological malignant characteristics harbored papillary features, the rates of recurrence-free survival were not affected. ${ }^{11}$ These findings suggest that rhabdoid and papillary features implicate morphological changes rather than anaplastic features in some cases.

\section{Lessons}

Rhabdoid meningiomas or post-traumatic meningiomas are rare clinical entities in the pediatric population. They are characterized by aggressive features such as rapid growth, invasion into the adjacent parenchyma, and frequent recurrence. Our findings suggest that traumatic damage to the arachnoid cells during childhood can prompt the development of a meningioma with rhabdoid morphology with an aggressive histological translation. To the best of our knowledge, pediatric meningiomas with rhabdoid features occurring after head trauma have not been published in English-language medical journals. The diagnosis needs to be made carefully, and close clinical follow-up is recommended.

\section{References}

1. Kotecha RS, Pascoe EM, Rushing EJ, et al. Meningiomas in children and adolescents: a meta-analysis of individual patient data. Lancet Oncol. 2011;12(13):1229-1239.

2. Cushing $H$, Eisenhardt $L$. Meningiomas. Their classification, regional behaviour, life history, and surgical end results. Bull Med Libr Assoc. 1938;27(2):185.
3. Annegers JF, Laws ER Jr, Kurland LT, Grabow JD. Head trauma and subsequent brain tumors. Neurosurgery. 1979;4(3):203-206.

4. Preston-Martin S, Pogoda JM, Schlehofer B, et al. An international case-control study of adult glioma and meningioma: the role of head trauma. Int J Epidemiol. 1998;27(4):579-586.

5. Barnett GH, Chou SM, Bay JW. Posttraumatic intracranial meningioma: a case report and review of the literature. Neurosurgery. 1986;18(1):75-78.

6. Artico M, Cervoni L, Carloia S, Palatinsky E, Delfini R. Development of intracranial meningiomas at the site of cranial fractures. Remarks on 15 cases. Acta Neurochir (Wien). 1995;136(3-4):132-134.

7. Caroli E, Salvati M, Rocchi G, Frati A, Cimatti M, Raco A. Post-traumatic intracranial meningiomas. Tumori. 2003;89(1):6-8.

8. François $P$, N'dri D, Bergemer-Fouquet AM, et al. Post-traumatic meningioma: three case reports of this rare condition and a review of the literature. Acta Neurochir (Wien). 2010;152(10):1755-1760.

9. Kepes JJ, Moral LA, Wilkinson SB, Abdullah A, Llena JF. Rhabdoid transformation of tumor cells in meningiomas: a histologic indication of increased proliferative activity: report of four cases. Am J Surg Pathol. 1998;22(2):231-238.

10. Perry A, Scheithauer BW, Stafford SL, Abell-Aleff PC, Meyer FB. "Rhabdoid" meningioma: an aggressive variant. Am J Surg Pathol. 1998;22(12):1482-1490.

11. Vaubel RA, Chen SG, Raleigh DR, et al. Meningiomas with rhabdoid features lacking other histologic features of malignancy: a study of 44 cases and review of the literature. J Neuropathol Exp Neurol. 2016;75(1):44-52.

12. Grossbach AJ, Mahaney KB, Menezes AH. Pediatric meningiomas: 65 -year experience at a single institution. J Neurosurg Pediatr. 2017;20(1):42-50.

13. Huntoon K, Pluto CP, Ruess L, et al. Sporadic pediatric meningiomas: a neuroradiological and neuropathological study of 15 cases. J Neurosurg Pediatr. 2017;20(2):141-148.

14. Buccoliero AM, Castiglione F, Rossi Degl'Innocenti D, et al. Pediatric rhabdoid meningioma: a morphological, immunohistochemical, ultrastructural and molecular case study. Neuropathology. 2011;31(1):59-65.

15. Wu YT, Ho JT, Lin YJ, Lin JW. Rhabdoid papillary meningioma: a clinicopathologic case series study. Neuropathology. 2011;31(6):599-605.

\section{Disclosures}

The authors report no conflict of interest concerning the materials or methods used in this study or the findings specified in this paper.

\section{Author Contributions}

Conception and design: Hayashi, Takata, Tachibana. Acquisition of data: Takata, Tamase. Analysis and interpretation of data: Hayashi, Takata, Sato. Drafting the article: Hayashi, Takata, Tachibana. Critically revising the article: Hayashi, Takata, Tachibana. Reviewed submitted version of manuscript: Hayashi, Takata, Tachibana, lizuka. Approved the final version of the manuscript on behalf of all authors: Hayashi. Statistical analysis: Takata. Administrative/technical/material support: Takata, Tachibana. Study supervision: Hayashi, Takata, lizuka. Pathological diagnosis: Sato.

\section{Correspondence}

Yasuhiko Hayashi: Kanazawa Medical University, Ishikawa, Japan. yhayashi@kanazawa-med.ac.jp. 